\title{
Coordinated Output Regulation of Multiple Heterogeneous Linear Systems
}

\author{
Ziyang Meng, Tao Yang, Dimos V. Dimarogonas, and Karl H. Johansson
}

\begin{abstract}
The coordinated output regulation problem for multiple heterogeneous linear systems is studied in this paper. Each agent is modeled as a linear multiple-input multipleoutput (MIMO) system with an exogenous input which represents the individual tracking objective for the agent. The multi-agent system as a whole has a group exogenous input which represents the tracking objective for the whole group. Under the constraints that the group exogenous input is only locally available to each agent and that the agents have only access to their neighbors' information, we propose an observerbased feedback controller to solve the coordinated output regulation problem. A high-gain approach is introduced and the information interactions are allowed to be switching over a finite set of fixed networks containing both graphs having a spanning tree and graphs that do not. A lower bound of the high gain parameters is explicitly given. It describes a fundamental relationship between the information interactions, the dwell time, the non-identical dynamics of different agents, and the high gain parameters.
\end{abstract}

\section{INTRODUCTION}

Coordinated control of multi-agent systems has recently drawn large attention due to its broad applications in physical, biological, social, and mechanical systems [1]-[3]. Motivated by the idea of using local information interactions to realize a global emergence [4]-[6], coordination of multiple linear dynamic systems is an interesting and fruitful research direction for the control community. For example, the authors of [7], [8] generalize the existing works on coordination of multiple single-integrator systems to the case of multiple linear time-invariant single-input systems. For a network of neutrally stable systems and polynomially unstable systems, the author of [9] proposes a design scheme for achieving synchronization. The case of switching communication topologies is considered in [10] and a consensustype observer is proposed to guarantee leaderless synchronization of multiple identical linear dynamic systems under a jointly connected communication topology. Similar problems are also considered in [11] and [12], where a frequently connected communication topology is studied in [11] and an assumption on the neutral stability is imposed in [12]. The authors of [13] propose a neighbor-based observer to solve the output synchronization problem for general linear time-invariant systems. An individual-based observer and a low-gain technique are used in [14] to synchronize a group

The authors are with ACCESS Linnaeus Centre, School of Electrical Engineering, Royal Institute of Technology, Stockholm 10044, Sweden. Email: $\{$ ziyangm, taoyang, dimos, kallej\}@kth.se. Corresponding author: Z. Meng. Tel. +46-722-839377.

This work has been supported in part by the Knut and Alice Wallenberg Foundation and the Swedish Research Council, and in part by EU HYCON 2 NoE. of linear systems with open-loop poles at most polynomially unstable. In addition, the classical Laplacian matrix is generalized in [15] to a so-called interaction matrix. A D-scaling approach is then used to stabilize this interaction matrix under both fixed and switching communication topologies. Synchronization of multiple heterogeneous linear systems has been investigated under both fixed and switching communication topologies [16], [17]. A similar problem is studied in [18], where a high-gain approach is proposed to dominate the non-identical dynamics of the agents. The cases of frequently connected and jointly connected communication topologies are studied in [19] and [20], respectively, where a slow switching condition and a fast switching condition are presented. More recently, the generalizations of coordination of multiple linear dynamic systems to the cooperative output regulation problem were studied in [21]-[23].

In this paper, we generalize the classical output regulation problem of an individual linear dynamic system to the coordinated output regulation problem of multiple heterogeneous linear dynamic systems. We consider the situation when each agent has an individual tracking objective and simultaneously there is a group tracking objective. The individual objective and the group objective are generated by autonomous systems (i.e., systems without inputs). Each individual objective is available to its corresponding agent while the group objective can be obtained only through constrained communication among the agents, i.e., the group objective corresponds to only a subset of the agents. Our goal is to find an observer-based feedback controller for each agent such that the output of each agent converges to a given trajectory determined by both the individual objective and the group objective. The contributions of this paper are threefold. First, we consider general linear dynamics, where the open-loop poles of the agents can be exponentially unstable and the dynamics are allowed to be different both with respect to dimensions and parameters. This relaxes the common assumption of identical dynamics [9], [10], [12], [13], [19] or open-loop poles at most polynomially unstable [10], [12], [17], [22]-[24]. Second, the information interaction can be switching from a graph set containing both a directed spanning tree set and a disconnected graph set. This extends the existing works on the case of fixed communication topologies [9], [13], [18], [21]. Third, an explicit lower bound on the high gain parameter is derived. The relationships between the dwell time [25], [26], the nonidentical dynamics among different agents and the high-gain parameters are explicitly given.

The remainder of the paper is organized as follows. In 
Section II, we give some basic definitions on network model. In Section III, we formulate the problem of coordinated output regulation of multiple heterogenous linear systems. The main results are presented in Section IV. A brief concluding remark is given in Section V.

\section{Network Model}

Graph theory is introduced to model the communication topology among agents. A directed graph $\mathbf{G}$ is defined as $\mathbf{G}:=(\mathbf{V}, \mathbf{E})$, where $\mathbf{V}=\left\{\nu_{1}, \nu_{2}, \ldots, \nu_{n}\right\}$ is the set of nodes and $\mathbf{E} \subseteq \mathbf{V} \times \mathbf{V}$ is a set of ordered pairs of nodes. We use the edge $\left(\nu_{j}, \nu_{i}\right)$ to denote that node $\nu_{i}$ can obtain information from node $\nu_{j}$. Here $\nu_{i}$ is the parent node and $\nu_{j}$ is the child node. All neighbors of node $\nu_{i}$ are defined by $N_{i}:=\left\{\nu_{j} \mid\left(\nu_{j}, \nu_{i}\right) \in \mathbf{E}\right\}$. A directed path is a sequence of edges of $\left(\nu_{i}, \nu_{j}\right),\left(\nu_{j}, \nu_{k}\right), \ldots$ A directed tree is defined as a directed graph, where every node has exactly one parent except for one node (this node has no parent and called the root), and the root has a directed path to every other node. A directed graph has a directed spanning tree if there contains at least one node having a directed path to all other nodes.

For a leader-follower graph $\overline{\mathbf{G}}:=(\overline{\mathbf{V}}, \overline{\mathbf{E}})$, we have $\overline{\mathbf{V}}=\left\{\nu_{0}, \nu_{1}, \ldots, \nu_{n}\right\}, \overline{\mathbf{E}} \subseteq \overline{\mathbf{V}} \times \overline{\mathbf{V}}$, where $\nu_{0}$ is the leader and $\nu_{1}, \nu_{2}, \ldots, \nu_{n}$ denote the followers. The leader-follower adjacency matrix $\overline{\mathbf{A}}=\left[a_{i j}\right] \in \mathbb{R}^{(n+1) \times(n+1)}$ is defined such that $a_{i j}$ is positive if $\left(\nu_{j}, \nu_{i}\right) \in \overline{\mathbf{E}}$ while $a_{i j}=0$ otherwise. Here we assume that $a_{i i}=0, i=0,1, \ldots, n$, and the leader has no parent, i.e.,, $a_{0 j}=0, j=0,1, \cdots, n$. The leaderfollower "grounded" Laplacian matrix $\overline{\mathbf{L}}=\left[l_{i j}\right] \in \mathbb{R}^{n \times n}$ associated with $\overline{\mathbf{A}}$ is defined as $l_{i i}=\sum_{j=0}^{n} a_{i j}$ and $l_{i j}=$ $-a_{i j}$, where $i \neq j$.

In this paper, we assume that the leader-follower communication topology $\overline{\mathbf{G}}_{\sigma(t)}$ is time-varying and switching from a finite set $\left\{\overline{\mathbf{G}}_{k}\right\}_{k \in \Upsilon}$, where $\Upsilon=\{1,2, \ldots, \delta\}$ is an index set and $\delta \in \mathbb{N}$ indicates its size. We impose the technical condition that $\overline{\mathbf{G}}_{\sigma(t)}$ is right-continuous, i.e., $\overline{\mathbf{G}}_{\sigma(t)}$ remains constant for $t \in\left[t_{l}, t_{l+1}\right), l=0,1, \ldots$ and switches at $t=t_{l}, l=1,2, \ldots$ In addition, we assume that $\inf _{l}\left(t_{l+1}-t_{l}\right) \geq \tau_{d}>0, l=0,1, \ldots$, where $\tau_{d}$ is a constant known as the dwell time [25].

Let the sets $\left\{\overline{\mathbf{A}}_{k}\right\}_{k \in \Upsilon}$ and $\left\{\overline{\mathbf{L}}_{k}\right\}_{k \in \Upsilon}$ be the leader-follower adjacency matrices and leader-follower grounded Laplacian matrices associated with $\left\{\overline{\mathbf{G}}_{k}\right\}_{k \in \Upsilon}$, respectively. Consequently, the time-varying leader-follower adjacency matrix and time-varying leader-follower grounded Laplacian matrix are defined as $\overline{\mathbf{A}}_{\sigma(t)}=\left[a_{i j}(t)\right]$ and $\overline{\mathbf{L}}_{\sigma(t)}=\left[l_{i j}(t)\right]$, where $\sigma:\left[t_{0}, \infty\right) \rightarrow \Upsilon$ is a piecewise constant function of time.

\section{PRoblem Formulation}

\section{A. Agent Dynamics}

Suppose that we have $n$ agents modeled by the linear MIMO systems with individual exogenous inputs:

$$
\begin{gathered}
\dot{x}_{i}=A_{i} x_{i}+B_{i} u_{i}, \\
\dot{\omega}_{i}=S_{i} \omega_{i},
\end{gathered}
$$

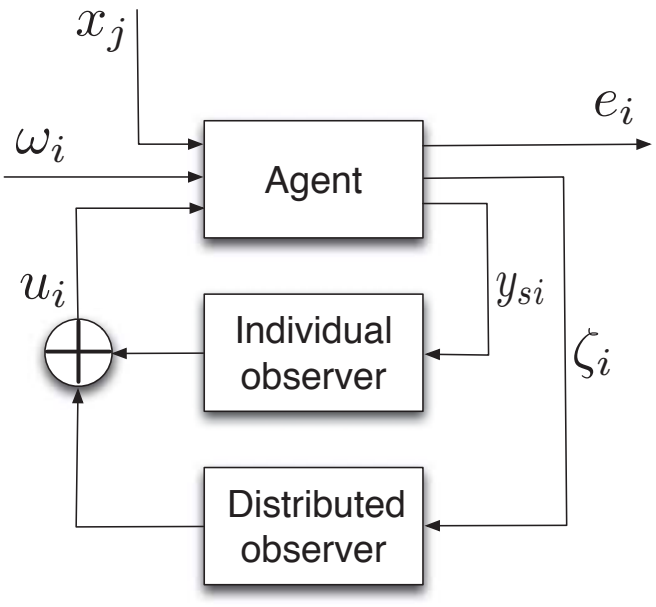

Fig. 1. Control architecture for agent $\nu_{i}$

where $x_{i} \in \mathbb{R}^{n_{i}}$ is the agent state, $\omega_{i} \in \mathbb{R}^{q_{i}}$ is the individual exogenous input, $u_{i} \in \mathbb{R}^{m_{i}}$ is the control input, $A_{i} \in \mathbb{R}^{n_{i} \times n_{i}}, B_{i} \in \mathbb{R}^{n_{i} \times m_{i}}, S_{i} \in \mathbb{R}^{q_{i} \times q_{i}}$.

Assume that there is a group exogenous input for the multi-agent system as a whole:

$$
\begin{aligned}
& \dot{x}_{0}=A_{0} x_{0}, \\
& y_{0}=C_{0} x_{0},
\end{aligned}
$$

where $x_{0} \in \mathbb{R}^{n_{0}}$ is the state, $y_{0} \in \mathbb{R}^{p}$ is the output, $A_{0} \in$ $\mathbb{R}^{m_{0} \times m_{0}}$, and $C_{0} \in \mathbb{R}^{p \times n_{0}}$.

\section{B. Control Architecture}

The control of each agent is supposed to have the structure shown in Fig. 1. More specifically, for the individual exogenous input tracking, available output information for agent $i$ is

$$
y_{s i}=C_{s i} x_{i}+D_{s i} \omega_{i},
$$

where $C_{s i} \in \mathbb{R}^{p \times n_{i}}$, and $D_{s i} \in \mathbb{R}^{p \times q_{i}}$.

For the group exogenous input tracking, only neighborbased output information is available due to the constrained communication. This means that not all the agents have access to $y_{0}$. The available information is the neighborbased sum of each agent's own output relative to that of its' neighbors, i.e.,

$$
\zeta_{i}=\sum_{j=0}^{n} a_{i j}(t)\left(y_{d i}-y_{d j}\right)
$$

is available for each agent $\nu_{i}$, where $a_{i j}(t), i=0,1, \ldots, n$, $j=0,1, \ldots, n$, is entry $(i, j)$ of the adjacency matrix $\overline{\mathbf{A}}_{\sigma(t)}$ associated with $\overline{\mathbf{G}}_{\sigma(t)}$ defined in Section II at time $t, y_{d i}$ can be represented by $y_{d i}=C_{d i} x_{i}, i=1,2, \ldots, n, y_{d 0}=y_{0}$. Also, the relative estimation information is available using the same communication topologies, i.e.,

$$
\widehat{\zeta}_{i}=\sum_{j=0}^{n} a_{i j}(t)\left(\widehat{y}_{i}-\widehat{y}_{j}\right)
$$




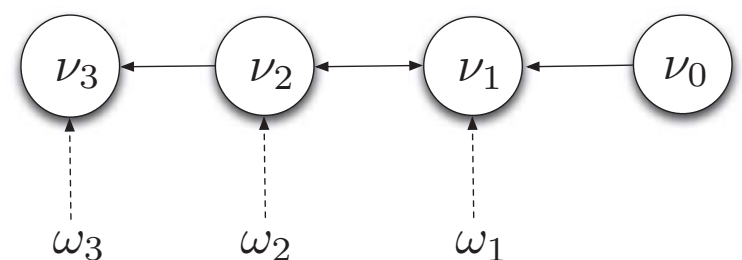

Fig. 2. Information flow associated with three agents $\nu_{1}, \nu_{2}, \nu_{3}$, the exogenous inputs $\omega_{1}, \omega_{2}, \omega_{3}$, and the group exogenous input $\nu_{0}$

is available for each agent $\nu_{i}$, where $\widehat{y}_{i}$ is an estimation produced internally by each agent $\nu_{i}$.

Fig. 2 gives an example of information flow among the agents and the group exogenous input $\nu_{0}$ for $n=3$ agents.

\section{Control Objective}

It is quite possible that there exist conflicting goals between each agent and the whole group in certain applications. Therefore, in this paper, the control objective of each agent is to track the group reference $x_{0}$ while following the individual reference $\omega_{i}, i=1,2, \ldots, n$. The tradeoff of these conflicting goals is captured by the coordinated output regulation tracking error (i.e., the total tracking error for both individual tracking and group tracking of each agent):

$$
e_{i}=C_{e i} x_{i}+D_{e i} \omega_{i}+D_{e 0} x_{0} .
$$

Note that it is possible that $C_{s i}, C_{d i}$, and $C_{e i}$ are the same observation matrices. Thus, our objective is to guarantee both individual objective asymptotic tracking and group asymptotic objective tracking, i.e., $\lim _{t \rightarrow \infty} e_{i}(t)=0$. We design an observer-based controller with available individual output information and neighbor-based group output information to solve this problem.

For the system shown in Fig. 2, the overall control can correspond to a leader-follower tracking problem, where the leader $x_{0}$ defines the group tracking objective, and $\omega_{i}$ describes the deviation between each agent and the leader.

\section{Main Result}

Before presenting the main result, we first impose some assumptions on the structure of the systems and information interactions.

Assumption 4.1. - $\left(A_{i}, B_{i}\right)$ is stabilizable, $i=1, \ldots, n$.

- The pair $\left[\begin{array}{ll}C_{d i} & -C_{0}\end{array}\right],\left[\begin{array}{cc}A_{i} & 0 \\ 0 & A_{0}\end{array}\right], i=1,2, \ldots, n$ is observable.

- $\left(S_{i}, D_{s i}\right), i=1,2, \ldots, n$ is observable,

For the communication topology set $\left\{\overline{\mathbf{G}}_{k}\right\}_{k \in \Upsilon}$, we assume that $\overline{\mathbf{G}}_{k}, \forall k \in \Upsilon_{c}$ is a graph containing a directed spanning tree with $\nu_{0}$ rooted. Without loss of generality, we relabel $\Upsilon_{c}:=\left\{1,2, \ldots, \delta_{1}\right\} \quad\left(1 \leq \delta_{1} \leq \delta\right)$, where $\delta_{1} \in \mathbb{N}$. The remaining graphs are labeled as $\overline{\mathbf{G}}_{k}, \forall k \in \Upsilon_{d}$, where $\Upsilon_{d}:=\left\{\delta_{1}+1, \delta_{1}+2, \ldots, \delta\right\}$. Denote the graph set $\overline{\mathbb{G}}_{c}=$ $\left\{\overline{\mathbf{G}}_{k}\right\}_{k \in \Upsilon_{c}}$ and the graph set $\overline{\mathbb{G}}_{d}=\left\{\overline{\mathbf{G}}_{k}\right\}_{k \in \Upsilon_{d}}$, respectively. We also denote $T^{d}(t)$ and $T^{c}(t)$ the total activation time when $\overline{\mathbf{G}}_{\sigma(\varsigma)} \in \overline{\mathbb{G}}_{d}$ and total activation time when $\overline{\mathbf{G}}_{\sigma(\varsigma)} \in \overline{\mathbb{G}}_{c}$ during $\varsigma \in\left[t_{0}, t\right)$. More specifically, define $z \in\{0,1, \ldots\}$ as the positive integer satisfying $t_{z} \leq t<t_{z+1}$, where $t_{z}$ is a switching instant. Also, define sets $\mathbb{K}_{c}(t)=\{\ell \mid \sigma(t) \in$ $\left.\Gamma_{c}, \forall t \in\left[t_{\ell}, t_{\ell+1}\right), \ell=0,1, \ldots, z\right\}$ and $\mathbb{K}_{d}(t)=\{\ell \mid \sigma(t) \in$ $\left.\Gamma_{d}, \forall t \in\left[t_{\ell}, t_{\ell+1}\right), \quad \ell=0,1, \ldots, z\right]$. Then, $T^{c}(t)=$ $\sum_{\forall \ell \in \mathbb{K}_{c}}\left(t_{\ell+1}-t_{\ell}\right)$ and $T^{d}(t)=t-t_{0}-T^{c}(t)$ if $z \in \mathbb{K}_{d}$ or $T^{d}(t)=\sum_{\forall \ell \in \mathbb{K}_{d}}\left(t_{\ell+1}-t_{\ell}\right)$ and $T^{c}(t)=t-t_{0}-T^{d}(t)$ if $z \in \mathbb{K}_{c}$.

Assumption 4.2. $\inf _{t \geq t_{0}} \frac{T^{c}(t)}{T^{d}(t)} \geq \kappa$, where $\kappa$ is a positive constant to be determined.

Remark 4.1. Assumption 4.2 implies that $\overline{\mathbb{G}}_{c}$ is non-empty and there exists a $T>0$, such that for any $t \geq t_{0}$, the switching signal $\sigma(t)$ satisfies $\left\{t \mid \overline{\mathbf{G}}_{\sigma(t)} \in \overline{\mathbb{G}}_{c}\right\} \cap[t, t+T] \neq \phi$. This condition means that the communication topology that contains a directed spanning tree need to come out frequently enough [11].

Assumption 4.3. The dwell time $\tau_{d}$ is a positive constant.

As suggested by Fig. 1, the design procedure to solve the coordinated output regulation problem includes three main parts: the first one is the distributed observer design for the group exogenous input, the second one is the individual observer design for the individual exogenous input, and the third one is the state-feedback control design. We present the design procedure in detail next.

\section{A. Distributed Observer Design for the Group Exogenous Input}

\section{Step 1: Pseudo-identical Linear Transformation}

Denote $\bar{x}_{i}=\left[x_{i}^{\mathrm{T}}, x_{0}^{\mathrm{T}}\right]^{\mathrm{T}}$. Then, (1a) and (2a) can be written by

$$
\begin{aligned}
\dot{\bar{x}}_{i} & =\bar{A}_{i} \bar{x}_{i}+\bar{B}_{i} u_{i}, \\
y_{d i}-y_{d 0} & =\bar{C}_{i} \bar{x}_{i}, \quad i=1,2, \ldots, n,
\end{aligned}
$$

where $\bar{A}_{i}=\left[\begin{array}{cc}A_{i} & 0 \\ 0 & A_{0}\end{array}\right] \in \mathbb{R}^{\bar{n}_{i} \times \bar{n}_{i}}, \bar{n}_{i}=n_{i}+n_{0}, \bar{B}_{i}=$ $\left[\begin{array}{c}B_{i} \\ 0\end{array}\right] \in \mathbb{R}^{\bar{n}_{i} \times m_{i}}, \bar{C}_{i}=\left[\begin{array}{ll}C_{d i} & -C_{0}\end{array}\right] \in \mathbb{R}^{p \times \bar{n}_{i}}$. Define $\chi_{i}=T_{i} \bar{x}_{i} \in \mathbb{R}^{p \bar{n}}, i=1,2, \ldots, n$, and $\bar{n}=\max _{i=1,2, \ldots, n} \bar{n}_{i}$, where

$$
T_{i}=\left[\begin{array}{c}
\bar{C}_{i} \\
\vdots \\
\bar{C}_{i} \bar{A}_{i}^{\bar{n}-1}
\end{array}\right] .
$$

Note that $T_{i}$ may be not a square matrix, but $T_{i}$ is full column rank since the pair $\left(\bar{C}_{i}, \bar{A}_{i}\right), i=1,2, \ldots, n$ is observable from Assumption 4.1. This implies that $T_{i}^{\mathrm{T}} T_{i}$ is nonsingular. Therefore, it follows that

$$
\begin{gathered}
\dot{\chi}_{i}=\left(\mathcal{A}+\mathcal{L}_{i}\right) \chi_{i}+\mathcal{B}_{i} u_{i}, \\
y_{d i}-y_{d 0}=\mathcal{C} \chi_{i}, \quad i=1,2, \ldots, n,
\end{gathered}
$$

where $\mathcal{A}=\left[\begin{array}{cc}0 & I_{p(\bar{n}-1)} \\ 0 & 0\end{array}\right] \in \mathbb{R}^{p \bar{n} \times p \bar{n}}, \mathcal{L}_{i}=\left[\begin{array}{c}0 \\ \Psi_{i}\end{array}\right]$, $\mathcal{B}_{i}=T_{i} \bar{B}_{i}, \mathcal{C}=\left[\begin{array}{ll}I_{p} & 0\end{array}\right] \in \mathbb{R}^{p \times p \bar{n}}$ for some matrix $\Psi_{i} \in \mathbb{R}^{p \times p \bar{n}}$. 


\section{Step 2: Distributed Observer Design}

Based on the neighbor-based group output information $\zeta_{i}$ and $\widehat{\zeta}_{i}$, the distributed observer is proposed for (5) as

$$
\begin{gathered}
\dot{\hat{\chi}}_{i}=\left(\mathcal{A}+\mathcal{L}_{i}\right) \widehat{\chi}_{i}+\mathcal{B}_{i} u_{i}+S(\varepsilon) \mathcal{P} \mathcal{C}^{\mathrm{T}} \\
\times\left(\sum_{j=0}^{n} a_{i j}(t)\left(y_{d i}-y_{d j}\right)-\sum_{j=0}^{n} a_{i j}(t)\left(\widehat{y}_{i}-\widehat{y}_{j}\right)\right) \\
i=1,2 \ldots, n,
\end{gathered}
$$

where $a_{i j}(t), i=0,1, \ldots, n, j=0,1, \ldots, n$, is entry $(i, j)$ of the adjacency matrix $\overline{\mathbf{A}}_{\sigma(t)}$ associated with $\overline{\mathbf{G}}_{\sigma(t)}$ defined in Section II at time $t, \widehat{y}_{i}=\mathcal{C} \widehat{\chi}_{i}, i=1,2, \ldots, n, \widehat{y}_{0}=0$. In addition, $S(\varepsilon)=\operatorname{diag}\left(I_{p} \varepsilon^{-1}, I_{p} \varepsilon^{-2}, \ldots, I_{p} \varepsilon^{-\bar{n}}\right)$, where $\varepsilon \in(0,1]$ is a positive constant to be determined, and $\mathcal{P}$ is a positive definite matrix satisfying

$$
\mathcal{A P}+\mathcal{P} \mathcal{A}^{\mathrm{T}}-2 \theta \mathcal{P} \mathcal{C}^{\mathrm{T}} \mathcal{C} \mathcal{P}+I_{p \bar{n}}=0,
$$

where $\theta=\min _{k \in \Upsilon_{c}} \beta_{k}$, and $\beta_{k}=\frac{1}{2} \lambda_{\min }\left(\overline{\mathbf{L}}_{k}+\overline{\mathbf{L}}_{k}^{\mathrm{T}}\right), \forall k \in$ $\Upsilon_{c}$.

Remark 4.2. We need the dynamics information $A_{0}$ and $C_{0}$ to construct the distributed observer (6) for each agent. But note that the output information $y_{0}$ is only available to a subset of the agents and the initial states of the group objective is not available.

Lemma 4.1. - All the eigenvalues of $\overline{\mathbf{L}}_{k}$ are in the closed right-half plane with those on the imaginary axis simple, where $\overline{\mathbf{L}}_{k}$ is associated with $\overline{\mathbf{G}}_{k}$ defined in Section II, and some $\overline{\mathbf{G}}_{k} \in\left\{\overline{\mathbf{G}}_{k}\right\}_{k \in \Upsilon}$.

- Furthermore, all the eigenvalues of $\overline{\mathbf{L}}_{k}$ are in the open right-half plane for $\overline{\mathbf{G}}_{k} \in\left\{\overline{\mathbf{G}}_{k}\right\}_{k \in \Upsilon_{c}}$.

Proof: See Theorems 4.25 and 4.29 in [27].

Lemma 4.2. Let Assumptions 4.1, 4.2, and 4.3 hold and choose $\kappa=\frac{\alpha+4 \theta \lambda_{\max }^{2}(\mathcal{P})}{1-\alpha}$, where $\alpha \in(0,1)$. Then, there exists an $\varepsilon^{*} \in(0,1]$ such that, if $\varepsilon \in\left(0, \varepsilon^{*}\right], \lim _{t \rightarrow \infty}\left(\chi_{i}(t)-\right.$ $\left.\widehat{\chi}_{i}(t)\right)=0, i=1,2 \ldots, n$, for system (5) and (6).

Proof: Define $\tilde{\chi}_{i}=\chi_{i}-\widehat{\chi}_{i}$. It then follows from (5) and (6) that

$$
\begin{gathered}
\dot{\tilde{\chi}}_{i}=\left(\mathcal{A}+\mathcal{L}_{i}\right) \widetilde{\chi}_{i}-S(\varepsilon) \mathcal{P C}^{\mathrm{T}}\left(\sum_{j=1}^{n} l_{i j}(t)\left(\left(y_{d j}-y_{d 0}\right)-\widehat{y}_{j}\right)\right), \\
i=1,2 \ldots, n,
\end{gathered}
$$

where $l_{i j}(t), i=1, \ldots, n, j=1, \ldots, n$, is the $(i, j)$ th entry of the adjacency matrix $\overline{\mathbf{A}}_{\sigma(t)}$ associated with $\overline{\mathbf{G}}_{\sigma(t)}$ defined in Section II at time $t$. It follows that

$$
\begin{array}{r}
\dot{\tilde{\chi}}_{i}=\left(\mathcal{A}+\mathcal{L}_{i}\right) \widetilde{\chi}_{i}-S(\varepsilon) \mathcal{P C}^{\mathrm{T}} \mathcal{C}\left(\sum_{j=1}^{n} l_{i j}(t)\left(\chi_{j}-\widehat{\chi}_{j}\right)\right), \\
i=1,2 \ldots, n .
\end{array}
$$

We then have that

$$
\dot{\tilde{\chi}}_{i}=\left(\mathcal{A}+\mathcal{L}_{i}\right) \tilde{\chi}_{i}-S(\varepsilon) \mathcal{P} \mathcal{C}^{\mathrm{T}} \mathcal{C}\left(\sum_{j=1}^{n} l_{i j}(t) \widetilde{\chi}_{j}\right),
$$

By introducing $\xi_{i}=\varepsilon^{-1} S^{-1}(\varepsilon) \tilde{\chi}_{i}$ and noticing that $\varepsilon S^{-1}(\varepsilon) \mathcal{A} S(\varepsilon)=\mathcal{A}$ and $\varepsilon \mathcal{C}^{\mathrm{T}} \mathcal{C} S(\varepsilon)=\mathcal{C}^{\mathrm{T}} \mathcal{C}$, we have that

$\varepsilon \dot{\xi}_{i}=\left(\mathcal{A}+\mathcal{L}_{i \varepsilon}\right) \xi_{i}-\mathcal{P} \mathcal{C}^{\mathrm{T}} \mathcal{C}\left(\sum_{j=1}^{n} l_{i j}(t) \xi_{j}\right), i=1,2 \ldots, n$, where $\mathcal{L}_{i \varepsilon}=\left[\begin{array}{c}0 \\ \varepsilon^{\bar{n}+1} \Psi_{i} S(\varepsilon)\end{array}\right]=O(\varepsilon)$. Define $\xi=$ $\left[\xi_{1}^{\mathrm{T}}, \xi_{2}^{\mathrm{T}}, \ldots, \xi_{n}^{\mathrm{T}}\right]^{\mathrm{T}}$ and $\mathcal{L}_{\varepsilon}=\operatorname{diag}\left(\mathcal{L}_{1 \varepsilon}, \mathcal{L}_{2 \varepsilon}, \ldots, \mathcal{L}_{n \varepsilon}\right)$. Then the overall dynamics can be written as

$$
\varepsilon \dot{\xi}=\left(I_{n} \otimes \mathcal{A}+\mathcal{L}_{\varepsilon}-\overline{\mathbf{L}}_{\sigma} \otimes\left(\mathcal{P C}^{\mathrm{T}} \mathcal{C}\right)\right) \xi
$$

where $\overline{\mathbf{L}}_{\sigma}$ is the Laplacian matrix defined in Section II.

Define piecewise Lyapunov function candidate $V_{k}=$ $\varepsilon \xi^{\mathrm{T}}\left(P_{k} \otimes \mathcal{P}^{-1}\right) \xi$, where $P_{k}$ is positive definite matrix satisfying

$$
\begin{gathered}
P_{k}\left(-\overline{\mathbf{L}}_{k}+\beta_{k} I_{n}\right)+\left(-\overline{\mathbf{L}}_{k}+\beta_{k} I_{n}\right)^{\mathrm{T}} P_{k}=-I_{n}<0, k \in \Upsilon_{c}, \\
P_{k}\left(-\overline{\mathbf{L}}_{k}\right)+\left(-\overline{\mathbf{L}}_{k}\right)^{\mathrm{T}} P_{k} \leq 0, \quad k \in \Upsilon_{d},
\end{gathered}
$$

where the second inequality is due to Lemma 4.1.

It then follows that

$$
\begin{aligned}
\dot{V}_{k} \leq & 2 \xi^{\mathrm{T}}\left(P_{k} \otimes \mathcal{P}^{-1} \mathcal{A}\right) \xi-2 \xi^{\mathrm{T}}\left(P_{k} \overline{\mathbf{L}}_{k} \otimes\left(\mathcal{C}^{\mathrm{T}} \mathcal{C}\right)\right) \xi \\
& +2 \xi^{\mathrm{T}}\left(P_{k} \otimes \mathcal{P}^{-1}\right) \mathcal{L}_{\varepsilon} \xi \\
\leq & \xi^{\mathrm{T}}\left(P_{k} \otimes\left(\mathcal{P}^{-1} \mathcal{A}+\mathcal{A}^{\mathrm{T}} \mathcal{P}^{-1}-2 \theta \mathcal{C}^{\mathrm{T}} \mathcal{C}\right)\right) \xi \\
& -\xi^{\mathrm{T}}\left(\left(2 P_{k} \overline{\mathbf{L}}_{k}-2 \theta P_{k}\right) \otimes\left(\mathcal{C}^{\mathrm{T}} \mathcal{C}\right)\right) \xi \\
& +2 \xi^{\mathrm{T}}\left(P_{k} \otimes \mathcal{P}^{-1}\right) \mathcal{L}_{\varepsilon} \xi \\
\leq & \xi^{\mathrm{T}}\left(P _ { k } \otimes \left(\mathcal{P}^{-1}\left(\mathcal{A P}+\mathcal{P} \mathcal{A}^{\mathrm{T}}-2 \theta \mathcal{P} \mathcal{C}^{\mathrm{T}} \mathcal{C} \mathcal{P}\right)\right.\right. \\
& \left.\left.\times \mathcal{P}^{-1}\right)\right) \xi-\xi^{\mathrm{T}}\left(\left(P_{k} \overline{\mathbf{L}}_{k}+\overline{\mathbf{L}}_{k}^{\mathrm{T}} P_{k}-2 \beta_{k} P_{k}\right)\right. \\
& \left.\otimes\left(\mathcal{C}^{\mathrm{T}} \mathcal{C}\right)\right) \xi+2 \lambda_{\max }\left(P_{k}\right) \lambda_{\max }\left(\mathcal{P}^{-1}\right)\left\|\mathcal{L}_{\varepsilon}\right\|\|\xi\|^{2} \\
\leq & -2 \xi^{\mathrm{T}}\left(P_{k} \otimes\left(\mathcal{P}^{-1} \mathcal{P}^{-1}\right)\right) \xi-\xi^{\mathrm{T}}\left(I_{n} \otimes\left(\mathcal{C}^{\mathrm{T}} \mathcal{C}\right)\right) \xi \\
& +\frac{2 \lambda_{\max }\left(P_{k}\right) \lambda_{\max }\left(\mathcal{P}^{-1}\right)\left\|\mathcal{L}_{\varepsilon}\right\|}{\varepsilon \lambda_{\min }\left(P_{k}\right) \lambda_{\min }\left(\mathcal{P}^{-1}\right)} V_{k} \\
\leq & -2 \xi^{\mathrm{T}}\left(P_{k} \otimes\left(\mathcal{P}^{-1} \mathcal{P}^{-1}\right)\right) \xi \\
& +\frac{2 \lambda_{\max }\left(P_{k}\right) \lambda_{\max }\left(\mathcal{P}^{-1}\right)\left\|\mathcal{L}_{\varepsilon}\right\|}{\varepsilon \lambda_{\min }\left(P_{k}\right) \lambda_{\min }\left(\mathcal{P}^{-1}\right)} V_{k}, \quad \forall k \in \Upsilon_{c}
\end{aligned}
$$

It follows that $\dot{V}_{k} \leq-\mu_{k} V_{k}, \forall k \in \Upsilon_{c}$, if $\left\|\mathcal{L}_{\varepsilon}\right\|<$ $\frac{\lambda_{\min }\left(P_{k}\right) \lambda_{\min }(\mathcal{P})}{4 \lambda_{\max }\left(P_{k}\right) \lambda_{\max }^{2}(\mathcal{P})}$, where $\mu_{k}=\frac{1}{2 \varepsilon \lambda_{\max }(\mathcal{P})}, \forall k \in \Upsilon_{c}$. Also, 
we have that

$$
\begin{aligned}
\dot{V}_{k} \leq & 2 \xi^{\mathrm{T}}\left(P_{k} \otimes\left(\mathcal{P}^{-1} \mathcal{A}\right)\right) \xi-2 \xi^{\mathrm{T}}\left(P_{k} \overline{\mathbf{L}}_{k} \otimes\left(\mathcal{C}^{\mathrm{T}} \mathcal{C}\right)\right) \xi \\
& +2 \xi^{\mathrm{T}}\left(P_{k} \otimes \mathcal{P}^{-1}\right) \mathcal{L}_{\varepsilon} \xi \\
\leq & \xi^{\mathrm{T}}\left(P_{k} \otimes\left(\mathcal{P}^{-1}\left(\mathcal{A P}+\mathcal{P} \mathcal{A}^{\mathrm{T}}\right) \mathcal{P}^{-1}\right)\right) \xi \\
& +2 \lambda_{\max }\left(P_{k}\right) \lambda_{\max }\left(\mathcal{P}^{-1}\right)\left\|\mathcal{L}_{\varepsilon}\right\|\|\xi\|^{2} \\
\leq & 2 \theta \xi^{\mathrm{T}}\left(P_{k} \otimes\left(\mathcal{C}^{\mathrm{T}} \mathcal{C}\right)\right) \xi-\frac{\lambda_{\min }\left(\mathcal{P}^{-1}\right)}{\varepsilon} V_{k} \\
& +\frac{2 \lambda_{\max }\left(P_{k}\right) \lambda_{\max }\left(\mathcal{P}^{-1}\right)\left\|\mathcal{L}_{\varepsilon}\right\|}{\varepsilon \lambda_{\min }\left(P_{k}\right) \lambda_{\min }\left(\mathcal{P}^{-1}\right)} V_{k}, \quad \forall k \in \Upsilon_{d} .
\end{aligned}
$$

It follows that $\dot{V}_{k} \leq-\mu_{k} V_{k}, \forall k \in \Upsilon_{d}$, if $\left\|\mathcal{L}_{\varepsilon}\right\|<$ $\frac{\lambda_{\min }\left(P_{k}\right) \lambda_{\min }(\mathcal{P})}{2 \lambda_{\max }\left(P_{k}\right) \lambda_{\max }^{2}(\mathcal{P})}$, where $\mu_{k}=\frac{2 \theta \lambda_{\max }(\mathcal{P})}{\varepsilon}, \forall k \in \Upsilon_{d}$.

Following the similar analysis of [25], [26], we let $t_{1}$, $t_{2}, \ldots$ be the time instants at which switching occurs and $\sigma=p_{j}$ on $\left[t_{j-1}, t_{j}\right)$. Then, for any $t$ satisfying $t_{0}<t_{1}<$ $\cdots<t_{\ell}<t<t_{\ell+1}$, define $V=\varepsilon \xi^{\mathrm{T}}\left(P_{\sigma(t)} \otimes \mathcal{P}^{-1}\right) \xi$ for (8). We have that, $\forall \varrho \in\left[t_{j-1}, t_{j}\right)$,

$$
\begin{aligned}
V(\varrho) & \leq e^{-\mu_{p_{j}}\left(\varrho-t_{j-1}\right)} V\left(t_{j-1}\right) \\
& \leq e^{-\mu^{c}\left(\varrho-t_{j-1}\right)} V\left(t_{j-1}\right), \quad p_{j} \in \Upsilon_{c}, \\
V(\varrho) & \leq e^{\mu_{p_{j}}\left(\varrho-t_{j-1}\right)} V\left(t_{j-1}\right) \\
& \leq e^{\mu^{d}\left(\varrho-t_{j-1}\right)} V\left(t_{j-1}\right), \quad
\end{aligned}
$$

where $\mu^{c}=\min _{k \in \Upsilon_{c}} \mu_{k}=\frac{1}{2 \varepsilon \lambda_{\max }(\mathcal{P})}$, $\mu^{d}=\max _{k \in \Upsilon_{d}} \mu_{k}=\frac{2 \theta \lambda_{\max }(\mathcal{P})}{\varepsilon}$. Define $a=$ $\frac{\lambda_{\max }(\mathcal{P})}{\lambda_{\min }(\mathcal{P})} \sup _{k, j \in \Upsilon} \frac{\lambda_{\max }\left(P_{k}\right)}{\lambda_{\min }\left(P_{j}\right)}$. We then know that $V\left(t_{j}\right) \leq a \lim _{t \uparrow t_{j}} V(t)$. Thus, it follows that

$$
V(t) \leq a^{\rho} e^{\mu^{d} T^{d}(t)-\mu^{c} T^{c}(t)} V\left(t_{0}\right),
$$

where $\rho$ denotes times of switching during $\left[t_{0}, t\right)$. Note that $\rho \leq \frac{t-t_{0}}{\tau_{d}}$. By choosing $\inf _{t \geq t_{0}} \frac{T^{c}(t)}{T^{d}(t)} \geq \kappa=\frac{\mu^{d}+\mu}{\mu^{c}-\mu}$ and some $\mu \in\left(0, \mu^{c}\right)$ for Assumption 4.2, we know that

$$
\begin{aligned}
V(t) & \leq a^{\rho} e^{-\mu\left(t-t_{0}\right)} V\left(t_{0}\right) \\
& \leq e^{\frac{t-t_{0}}{\tau_{d}} \ln a-\mu\left(t-t_{0}\right)} V\left(t_{0}\right) \\
& =e^{-\left(\mu-\frac{\ln a}{\tau_{d}}\right)\left(t-t_{0}\right)} V\left(t_{0}\right) .
\end{aligned}
$$

Furthermore, set $\mu=\alpha \mu^{c}$, where some $\alpha \in(0,1)$. We then have that $\kappa=\frac{\alpha+4 \theta \lambda_{\max }^{2}(\mathcal{P})}{1-\alpha}$, and

$$
V(t) \leq e^{-\left(\frac{\alpha}{2 \varepsilon \lambda_{\max }(\mathcal{P})}-\frac{\ln a}{\tau_{d}}\right)\left(t-t_{0}\right)} V\left(t_{0}\right) .
$$

It follows that if $\varepsilon<\frac{\alpha \tau_{d}}{2 \lambda_{\max }(\mathcal{P}) \ln a}$, we have for (8) that

$$
\|\xi(t)\| \leq c e^{-\frac{1}{2}\left(\frac{\alpha}{2 \varepsilon \lambda_{\max }(\mathcal{P})}-\frac{\ln a}{\tau_{d}}\right)\left(t-t_{0}\right)}\left\|\xi\left(t_{0}\right)\right\|,
$$

where $c=\sqrt{\frac{\lambda_{\max }(\mathcal{P}) \sup _{k \in \Upsilon} \lambda_{\max }\left(P_{k}\right)}{\lambda_{\min }(\mathcal{P}) \sup _{k \in \Upsilon} \lambda_{\min }\left(P_{k}\right)}}$.

Therefore, $\varepsilon^{*}$ is chosen satisfying $\varepsilon^{*}<\frac{\alpha \tau_{d}}{2 \lambda_{\max }(\mathcal{P}) \ln a}$ and $\left\|\mathcal{L}_{\mathcal{E}^{*}}\right\|<\inf _{k \in \Upsilon} \frac{\lambda_{\min }\left(P_{k}\right) \lambda_{\min }(\mathcal{P})}{4 \lambda_{\max }\left(P_{k}\right) \lambda_{\max }^{2}(\mathcal{P})}$. This implies that if $\varepsilon \in$ $\left(0, \varepsilon^{*}\right], \xi=0$ is a globally exponentially stable equilibrium of (8). This completes the proof.

\section{Step 3: Linear Inverse Transformation}

We have that

$$
\widehat{\bar{x}}_{i}=\left(T_{i}^{\mathrm{T}} T_{i}\right)^{-1} T_{i}^{\mathrm{T}} \widehat{\chi}_{i}=\left[\widehat{x}_{i}^{\mathrm{T}}, \widehat{x}_{0 i}^{\mathrm{T}}\right]^{\mathrm{T}}, \quad i=1,2, \ldots, n,
$$

which will be used in the control input design.

\section{B. Individual Observer Design for the Exogenous Input}

Based on the information of $\widehat{x}_{i}$ and the individual output information $y_{s i}$, the following individual observer for each agent is proposed

$$
\dot{\hat{\omega}}_{i}=S_{i} \widehat{\omega}_{i}+K_{s i}\left(C_{s i} \widehat{x}_{i}+D_{s i} \widehat{\omega}_{i}-y_{s i}\right), \quad i=1,2 \ldots, n .
$$

Lemma 4.3. Let that Assumptions 4.1, 4.2, and 4.3 hold. Then, (10) ensures that $\lim _{t \rightarrow \infty}\left(\omega_{i}(t)-\widehat{\omega}_{i}(t)\right)=0, i=$ $1,2 \ldots, n$, for system (1a), (1b), where $K_{s i}$ is chosen such that $S_{i}+K_{s i} D_{s i}$ is Hurwitz stable.

Proof: Define $\widetilde{\omega}_{i}=\omega_{i}-\widehat{\omega}_{i}$, we have that

$\dot{\tilde{\omega}}_{i}=S_{i} \widetilde{\omega}_{i}+K_{s i} D_{s i} \widetilde{\omega}_{i}+K_{s i} C_{s i}\left(\widehat{x}_{i}-x_{i}\right), \quad i=1,2 \ldots, n$.

Note that $\bar{x}_{i}=\left(T_{i}^{\mathrm{T}} T_{i}\right)^{-1} T_{i}^{\mathrm{T}} \chi_{i}=\left[x_{i}^{\mathrm{T}}, x_{0}^{\mathrm{T}}\right]^{\mathrm{T}}$. It thus follows from Lemma 4.2 and (9) that $\lim _{t \rightarrow \infty}\left(x_{i}(t)-\widehat{x}_{i}(t)\right)=$ 0 . Since $S_{i}+K_{s i} D_{s i}$ is Hurwitz stable, we know that $\lim _{t \rightarrow \infty}\left(\widehat{\omega}_{i}(t)-\omega_{i}(t)\right)=0$.

\section{Regulated State-feedback Control Input}

We now design a controller to regulate $e_{i}$ to zero for each agent based on the state information $x_{i}, \omega_{i}$, and $x_{0}$. Let $\Pi_{1 i}$, $\Pi_{2 i}, \Gamma_{1 i}$ and $\Gamma_{2 i}$ be the solutions of the following regulator equation

$$
\begin{gathered}
\Pi_{1 i} S_{i}=A_{i} \Pi_{1 i}+B_{i} \Gamma_{1 i}, \\
\Pi_{2 i} A_{0}=A_{i} \Pi_{2 i}+B_{i} \Gamma_{2 i}, \\
C_{e i} \Pi_{1 i}=D_{e i}, \\
C_{e i} \Pi_{2 i}=D_{e 0}, \quad i=1,2 \ldots, n .
\end{gathered}
$$

Lemma 4.4. Assume that Assumption 4.1 is satisfied. If the regulator equations (11) are solvable, the state-feedback controller $u_{i}=K_{i}\left(x_{i}-\Pi_{1 i} \omega_{i}-\Pi_{2 i} x_{0}\right)+\Gamma_{1 i} \omega_{i}+\Gamma_{2 i} x_{0}$ ensures that $\lim _{t \rightarrow \infty} e_{i}(t)=0, i=1,2 \ldots, n$, for the closed loop system (1) and (2), where $K_{i}$ is chosen such that $A_{i}+B_{i} K_{i}$ is Hurwitz.

Proof: Consider the following individual output regulation problem

$$
\begin{gathered}
\dot{x}_{i}=A_{i} x_{i}+B_{i} u_{i}, \\
\dot{\omega}_{i}=S_{i} \omega_{i}, \\
\dot{x}_{0}=A_{0} x_{0}, \\
e_{i}=C_{e i} x_{i}+D_{e i} \omega_{i}+D_{e 0} x_{0}, \quad i=1,2, \ldots, n .
\end{gathered}
$$

Using the result of classic output regulation [28], we know that

$$
u_{i}(t)=K_{i}\left(x_{i}-\Pi_{1 i} \omega_{i}-\Pi_{2 i} x_{0}\right)+\Gamma_{1 i} \omega_{i}+\Gamma_{2 i} x_{0},
$$

ensures that $\lim _{t \rightarrow \infty} e_{i}(t)=0, i=1,2 \ldots, n$, for the closed loop system (12), where $\Pi_{1 i}, \Pi_{2 i}, \Gamma_{1 i}$, and $\Gamma_{2 i}$ are the solutions of (11). 


\section{Main Result}

The observer-based controller is proposed as

$$
u_{i}=K_{i} \widehat{x}_{i}+\left(\Gamma_{1 i}-K_{i} \Pi_{1 i}\right) \widehat{\omega}_{i}+\left(\Gamma_{2 i}-K_{i} \Pi_{2 i}\right) \widehat{x}_{0 i},
$$

where $\Pi_{1 i}, \Pi_{2 i}, \Gamma_{1 i}$, and $\Gamma_{2 i}$ are the solutions of the regulator equation (11), $\widehat{x}_{i}$ and $\widehat{x}_{0 i}$ are given in (9) and produced in (6), and $\widehat{\omega}_{i}$ is given in (10).

Theorem 4.1. Let Assumptions 4.1, 4.2, and 4.3 hold and choose $\kappa=\frac{\alpha+4 \theta \lambda_{\max }^{2}(\mathcal{P})}{1-\alpha}$. Then, there exists $\varepsilon^{*} \in(0,1]$ such that, if $\varepsilon \in\left(0, \varepsilon^{*}\right]$, (14) ensures that $\lim _{t \rightarrow \infty} e_{i}(t)=0$, $i=1,2 \ldots, n$, for (1), (2), and (4).

Proof: Note that the closed-loop system (1) with (14) can be written by

$$
\begin{aligned}
\dot{x}_{i}= & A_{i} x_{i}+B_{i}\left(K_{i} x_{i}+\left(\Gamma_{1 i}-K_{i} \Pi_{1 i}\right) \omega_{i}+\left(\Gamma_{2 i}-K_{i} \Pi_{2 i}\right)\right. \\
& \left.\times x_{0}\right)+B_{i}\left(K_{i}\left(\widehat{x}_{i}-x_{i}\right)+\left(\Gamma_{1 i}-K_{i} \Pi_{1 i}\right)\left(\widehat{\omega}_{i}-\omega_{i}\right)\right. \\
& \left.+\left(\Gamma_{2 i}-K_{i} \Pi_{2 i}\right)\left(\widehat{x}_{0 i}-x_{0}\right)\right) .
\end{aligned}
$$

Therefore, it follows from Lemmas 4.2-4.4 and the separation principle that $\lim _{t \rightarrow \infty} e_{i}(t)=0, i=1,2 \ldots, n$.

\section{CONCLUSIONS}

This paper studied the coordinated output regulation problem of multiple heterogeneous linear systems. We first formulated the coordinated output regulation problem and specified the information that is available for each agent. A high-gain based distributed observer and an individual observer were introduced for each agent and an observerbased controller was designed to solve the problem. The information interactions among the agents and the group exogenous input were allowed to be switching over a finite set of fixed networks containing both the graph having a spanning tree and the graph having not. The relationship of the information interactions, the dwell time, the non-identical dynamics of different agents, and the high-gain parameters were explicitly given.

\section{REFERENCES}

[1] J. Cortes, S. Martinez, and F. Bullo, "Robust rendezvous for mobile autonomous agents via proximity graphs in arbitrary dimensions," IEEE Transactions on Automatic Control, vol. 51, no. 8, pp. 12891298, 2006.

[2] H. G. Tanner, A. Jadbabaie, and G. J. Pappas, "Flocking in fixed and switching networks," IEEE Transactions on Automatic Control, vol. 52, no. 5, pp. 863-868, 2007.

[3] N. Chopra and M. W. Spong, "On exponential synchronization of kuramoto oscillators," IEEE Transactions on Automatic Control, vol. 54, no. 2, pp. 353-357, 2009.

[4] T. Vicsek, A. Czirok, E. B. Jacob, I. Cohen, and O. Schochet, "Novel type of phase transitions in a system of self-driven particles," Physical Review Letters, vol. 75, no. 6, pp. 1226-1229, 1995.

[5] A. Jadbabaie, J. Lin, and A. S. Morse, "Coordination of groups of mobile autonomous agents using nearest neighbor rules," IEEE Transactions on Automatic Control, vol. 48, no. 6, pp. 988-1001, 2003.

[6] R. Olfati-Saber, J. A. Fax, and R. M. Murray, "Consensus and cooperation in networked multi-agent systems," Proceedings of the IEEE, vol. 95, no. 1, pp. 215-233, 2007.

[7] W. Ren, K. L. Moore, and Y. Chen, "High-order and model reference consensus algorithms in cooperative control of multivehicle systems," J. Dyn. Sys., Meas., Control, vol. 129, no. 5, pp. 678-688, 2007.
[8] P. Wieland, J.-S. Kim, and F. Allgower, "On topology and dynamics of consensus among linear high-order agents," International Journal of Systems Science, vol. 42, no. 10, pp. 1831-1842, 2011.

[9] S. E. Tuna, "Conditions for synchronizability in arrays of coupled linear systems," IEEE Transactions on Automatic Control, vol. 54, no. 10, pp. 2416-2420, 2009.

[10] L. Scardovi and R. Sepulchre, "Synchronization in networks of identical linear systems," Automatica, vol. 45, pp. 2557-2562, 2009.

[11] J. Wang, D. Cheng, and X. Hu, "Consensus of multi-agent linear dynamic systems," Asian Journal of Control, vol. 10, no. 2, pp. 144 155, 2008.

[12] W. Ni and D. Cheng, "Leader-following consensus of multi-agent systems under fixed and switching topologies," Systems and Control Letters, vol. 59, no. 3-4, pp. 209-217, 2010.

[13] Z. Li, Z. Duan, G. Chen, and L. Huang, "Consensus of multiagent systems and synchronization of complex networks: A unified viewpoint," IEEE Transactions on Circuits and Systems - I: Regular Papers vol. 57, no. 1, pp. 213-224, 2010.

[14] J. H. Seo, H. Shim, and J. Back, "Consensus of high-order linear systems using dynamic output feedback compensator: low gain approach,' Automatica, vol. 45, no. 11, pp. 2659-2664, 2009.

[15] T. Yang, S. Roy, Y. Wan, and A. Saberi, "Constructing consensus controllers for networks with identical general linear agents," International Journal of Robust and Nonlinear Control, vol. 21, no. 11, pp. 1237-1256, 2011

[16] J. Lunze, "Synchronization of heterogeneous agents," IEEE Trans. Automatic Control, vol. 57, no. 11, pp. 2885-2890, 2012.

[17] P. Wieland, R. Sepulchre, and F. Allgower, "An internal model principle is necessary and sufficient for linear output synchronization," Automatica, vol. 47, no. 5, pp. 1068-1074, 2011.

[18] H. F. Grip, T. Yang, A. Saberi, and A. A. Stoorvogel, "Output synchronization for heterogeneous networks of non-introspective agents," Automatica, vol. 48, pp. 2444-2453, 2012.

[19] D. Vengertsev, H. Kim, H. Shim, and J. H. Seo, "Consensus of output-coupled linear multi-agent systems under frequently connected network," in 49th IEEE Conference on Decision and Control, Hilton Atlanta Hotel, Atlanta, GA, USA, 2010, pp. 4559-4564.

[20] H. Kim, H. Shim, J. Back, and J. H. Seo, "Consensus of outputcoupled linear multi-agent systems under fast switching network: Averaging approach," Automatica, vol. 49, no. 1, pp. 267-272, 2013

[21] X. Wang, Y. Hong, J. Huang, and Z. Jiang, "A distributed control approach to a robust output regulation problem for multi-agent linear systems," IEEE Transactions on Automatic Control, vol. 55, no. 12 pp. 2891-2895, 2012.

[22] Y. Su and J. Huang, "Stability of a class of linear switching systems with applications to two consensus problems," IEEE Transactions on Automatic Control, vol. 57, no. 6, pp. 1420-1430, 2012.

[23] — "Cooperative output regulation with application to multi-agent consensus under switching network," IEEE Transactions on Systems, Man, Cybernetics, Part B: Cybernetics, vol. 42, no. 3, pp. 864-875, 2012

[24] - "Cooperative output regulation of linear multi-agent systems," IEEE Transactions on Automatic Control, vol. 57, no. 4, pp. 10621066,2012

[25] D. Liberzon and A. S. Morse, "Basic problems in stability and design of switched systems," IEEE Control Systems Magazine, vol. 19, pp. 59-70, 1999.

[26] G. Zhai, B. Hu, K. Yasuda, and A. N. Michel, "Piecewise Lyapunov functions for switched systems with average dwell time," Asian Journal of Control, vol. 2, no. 3, pp. 192-197, 2000.

[27] Z. Qu, Cooperative control of dynamical systems: applications to autonomous vehicles. Springer, 2009

[28] A. Saberi, A. A. Stoorvogel, and P. Sannuti, Control of linear systems with regulation and input constraints. Communications and Control Engineering. London, UK: Springer, 2003 